\title{
First direct observation of Dirac fermions in graphite
}

\author{
S. Y. ZHOU' ${ }^{1,2}$, G.-H. GWEON ${ }^{1}$, J. GRAF², A. V. FEDOROV 3 , C. D. SPATARU 1,4 , R. D. DIEHL ${ }^{5}$, \\ Y. KOPELEVICH' ${ }^{6}$, D.-H. LEE ${ }^{1,2}$, STEVEN G. LOUIE ${ }^{1,2}$ AND A. LANZARA',2* \\ ${ }^{1}$ Department of Physics, University of California, Berkeley, California 94720, USA \\ ${ }^{2}$ Materials Sciences Division, Lawrence Berkeley National Laboratory, Berkeley, California 94720, USA \\ ${ }^{3}$ Advanced Light Source, Lawrence Berkeley National Laboratory, Berkeley, California 94720, USA \\ ${ }^{4}$ Chemical Sciences Division, Lawrence Berkeley National Laboratory, Berkeley, California 94720, USA \\ ${ }^{5}$ Department of Physics and Materials Research Institute, Penn State University, University Park, Pennsylvania 16802, USA \\ ${ }^{6}$ Instituto de Física 'Gleb Wataghin', Universidade Estadual de Campinas, Unicamp 13083-970, Campinas, Sao Paulo, Brazil \\ *e-mail: ALanzara@lbl.gov
}

Published online: 27 August 2006; doi:10.1038/nphys393

0 riginating from relativistic quantum field theory, Dirac fermions have been invoked recently to explain various peculiar phenomena in condensed-matter physics, including the novel quantum Hall effect in graphene ${ }^{1,2}$, the magnetic-field-driven metal-insulator-like transition in graphite ${ }^{3,4}$, superfluidity in ${ }^{3} \mathrm{He}$ (ref. 5) and the exotic pseudogap phase of high-temperature superconductors ${ }^{6,7}$. Despite their proposed key role in those systems, direct experimental evidence of Dirac fermions has been limited. Here, we report the first direct observation of relativistic Dirac fermions with linear dispersion near the Brillouin zone (BZ) corner $\mathrm{H}$, which coexist with quasiparticles that have a parabolic dispersion near another $\mathrm{BZ}$ corner $\mathrm{K}$. In addition, we also report a large electron pocket that we attribute to defect-induced localized states. Thus, graphite presents a system in which massless Dirac fermions, quasiparticles with finite effective mass and defect states all contribute to the low-energy electronic dynamics.

For most condensed-matter systems, the physics is formulated in terms of the non-relativistic Schrödinger equation, and the lowenergy excitations are quasiparticles with finite effective mass. For some special systems, for example, graphene/graphite, where the dispersion is expected to be linear near the Fermi energy, $E_{\mathrm{F}}$, and only touch $E_{\mathrm{F}}$ at one point (Dirac point), the physics is described by the relativistic Dirac equation with the speed of light replaced by the Fermi velocity $v_{\mathrm{F}}$. The low-energy excitations in this case are Dirac fermions, which have zero effective mass and a vanishing density of states at the Dirac point. The Dirac fermions are proposed to be responsible for various anomalous phenomena observed in these systems ${ }^{1-3,8}$. So far, transport experiments in graphene have shown results in agreement with the presence of Dirac fermions ${ }^{1,2}$. Phase analysis of quantum oscillations in graphite has also suggested coexistence of both Dirac fermions and quasiparticles with finite effective mass ${ }^{9}$. Here, we report the first direct observation of massless Dirac fermions coexisting with quasiparticles with finite effective mass in graphite, by using angle-resolved photoemission spectroscopy (ARPES). ARPES provides the advantage of directly probing the electronic structure with both energy and momentum information, not accessible by any other measurement.

Figure 1a shows an ARPES intensity map measured near the Brillouin zone (BZ) corner $\mathrm{H}$. The out-of-plane momentum, $k_{z}$, is $0.5 c^{*}$, where $c^{*}$ is the reciprocal lattice constant (see the Methods section for the extraction of $k_{z}$ values). Following the maximum intensity in this map, a linear $\Lambda$-shaped dispersion can be clearly observed. The dispersion can be better extracted by following the peak positions in the momentum distribution curves (MDCs), momentum scans at constant energies, shown in Fig. 1b. Here, two peaks in the MDCs disperse linearly and merge near $E_{\mathrm{F}}$. The Fermi velocity extracted from the dispersion is $0.91 \pm 0.15 \times 10^{6} \mathrm{~m} \mathrm{~s}^{-1}$, similar to a value $1.1 \times 10^{6} \mathrm{~m} \mathrm{~s}^{-1}$ reported by a magnetoresistance study of graphene ${ }^{2}$. We note that at low energy near $E_{\mathrm{F}}$, this linear dispersion is also observed along other cuts through the $\mathrm{H}$ point, with similar Fermi velocity. This linear and isotropic dispersion is in agreement with the behaviour of Dirac fermions.

Another way of probing the linear and isotropic dispersion is to study the intensity maps at constant energy. At $E_{\mathrm{F}}$ (Fig. 2a), the intensity map shows a small object near $\mathrm{H}$. The details of this small object will be discussed later. With increasing binding energy, this object expands and shows a circular shape (Fig. 2b,c). We note that only the circular shape in the first BZ is clearly observed. This is attributed to the dipole matrix element ${ }^{10}$, which suppresses or enhances the intensity in different BZs. However, taking the three-fold symmetry of the sample, this circular shape in the first $\mathrm{BZ}$ is expected to extend to other BZs and thus the electronic structure is isotropic near $\mathrm{H}$. As the energy changes to $-0.9 \mathrm{eV}$, the constant energy map deviates slightly from the circular shape (see the arrow in Fig. 2d). This deviation increases with binding energy and a trigonal distortion is clearly observed at $-1.2 \mathrm{eV}$ (Fig. 2e). This trigonal distortion is determined by the relevant 

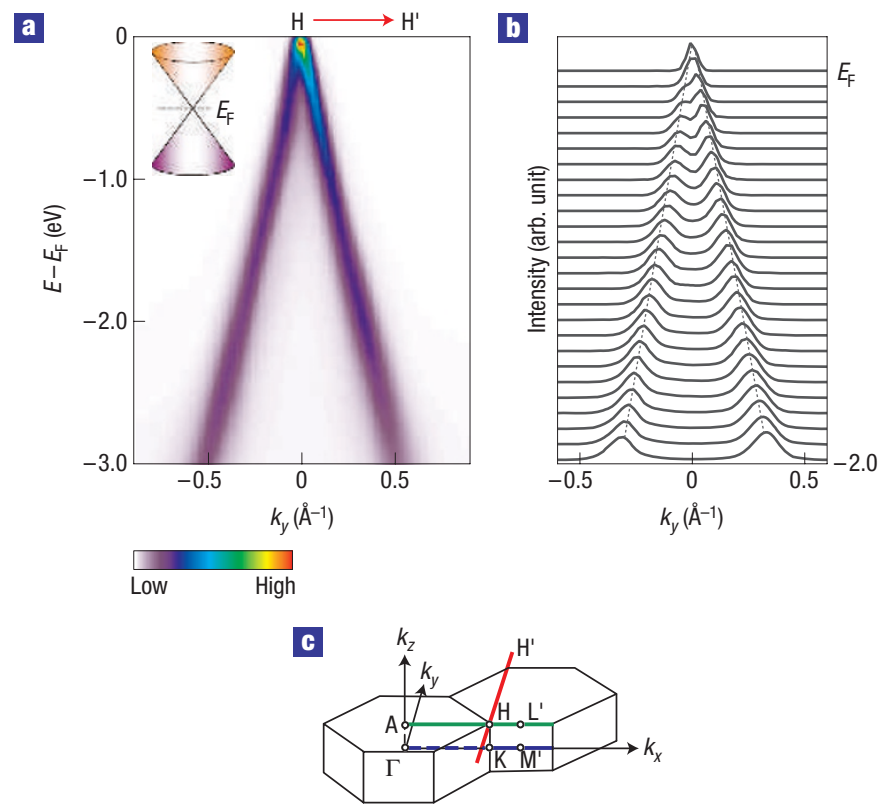

Figure 1 Linear $\boldsymbol{\Lambda}$-shaped dispersion near the BZ corner $\mathbf{H}$. a, ARPES intensity map taken near the $\mathrm{H}$ point (photon energy $h v=140 \mathrm{eV}, k_{z} \approx 0.50 c^{*}$ ), along a cut through $\mathrm{H}$ and perpendicular to $k_{x}$ (see the red line in the BZ shown in c). The inset shows a schematic diagram of the Dirac cone dispersion near $E_{\mathrm{F}}$ in the three-dimensional $E-k_{x}-k_{y}$ space. $\mathbf{b}, \mathrm{MDCs}$ from $E_{\mathrm{F}}$ to $-2.0 \mathrm{eV}$. The MDCs are normalized to have the same amplitude and are displaced by the same amount so that the dispersion can be directly viewed by following the peak positions at each energy. The dotted lines are guides to the eyes for the linear-dispersing peaks in the MDCs. c, Three-dimensional BZ for graphite with high-symmetry directions relevant for this paper highlighted with red, green and blue lines.

tight-binding parameters for graphite and further studies to analyse this trigonal distortion are in progress. Overall, Fig. 2 shows that from $E_{\mathrm{F}}$ to $-0.6 \mathrm{eV}$, the electronic structure is isotropic in the $k_{x}-k_{y}$ plane. Similarly, the Fermi velocity measured within the first BZ is $1.0 \times 10^{6} \mathrm{~m} \mathrm{~s}^{-1}$ with a $\leq 10 \%$ variation along different directions, consistent with the circular constant energy maps shown here. Combining the results of Figs 1 and 2, we conclude that from $E_{\mathrm{F}}$ to $-0.6 \mathrm{eV}$, the dispersion shows a cone-like behaviour near each BZ corner $\mathrm{H}$, similar to that expected for graphene (Fig. 2f).

To resolve the details of the low-energy dispersion and the small object at $E_{\mathrm{F}}$ (Fig. 2a), we show in Fig. 3a an intensity map measured near $\mathrm{H}$ with lower photon energy to give better energy and momentum resolution. In the intensity map, two bands dispersing linearly towards $E_{\mathrm{F}}$ can be distinguished, as is also clear in the MDCs (Fig. 3b) where two peaks can be observed for all binding energies. The extracted dispersion (open circles in Fig. 3a) from MDCs (Fig. 3b) shows two bands dispersing linearly towards $E_{\mathrm{F}}$, with a minimum separation of $0.020 \pm 0.004 \AA^{-1}$ at $E_{\mathrm{F}}$. This linear dispersion near the $\mathrm{H}$ point, as well as the isotropic electronic structure shown in Fig. 2 from $E_{\mathrm{F}}$ to $-0.6 \mathrm{eV}$, is a basic characteristic of Dirac quasiparticles, which points to the presence of Dirac quasiparticles in the low-energy excitations near the $\mathrm{H}$ point in graphite. Furthermore, from the extracted dispersions, the Dirac point is extrapolated to be $50 \pm 20 \mathrm{meV}$ above $E_{\mathrm{F}}$, and thus the small object observed at $E_{\mathrm{F}}$ is a hole pocket, in agreement with previous studies of the three-dimensional band structure of graphite $^{9,11}$. Assuming an ellipsoidal shape for the hole pocket ${ }^{12}$, the hole concentration is estimated to be $3.1 \pm 1.3 \times 10^{18} \mathrm{~cm}^{-3}$, from the $0.020 \AA^{-1}$ separation of the peaks at $E_{\mathrm{F}}$. This hole concentration is in agreement with reported values ${ }^{12-14}$. We note that, given the current resolution of the ARPES technique, we are not able to resolve the two hole pockets at the $\mathrm{H}$ point reported by the other experimental probe ${ }^{15}$. In fact, according to ref. 15 , the difference in energy between these two hole pockets at the $\mathrm{H}$ point is $\leq 1 \mathrm{meV}$, which is beyond the current resolution of the ARPES technique. The presence of holes with Dirac fermion dispersion is further supported by the angle-integrated intensity (Fig. 3c), which is proportional to the two-dimensional density of states, barring the matrix element. In this energy range, a linear behaviour, similar to that expected for Dirac fermions, is observed. In addition, the energy intersect is at $\approx 50 \mathrm{meV}$ above $E_{\mathrm{F}}$, in agreement with the Dirac point energy extrapolated from the dispersions.

Figures 1-3 show that near the $\mathrm{H}$ point, the low-energy excitations in graphite are Dirac fermions characterized by linear and isotropic cone-like dispersion, in agreement with transport measurements in graphite where Dirac fermions are suggested to coexist with quasiparticles that have finite effective mass ${ }^{9}$. To gain direct insight into the different types of quasiparticles, ARPES can provide a unique advantage by directly measuring the effective mass as well as accessing its momentum dependence. Figure 4a shows the intensity map near another high-symmetry point in the BZ corner, the K point. The dispersion (open circles) shows a parabolic behaviour, in sharp contrast to the linear behaviour observed near the $\mathrm{H}$ point (Figs 1,3). This parabolic dispersion points to the presence of quasiparticles with finite effective mass. To determine the effective mass, we first extract the low-energy dispersion, then fit the MDC and energy distribution curve (EDC) dispersions (Fig. 3b,c) with a parabolic function. In both cases, the effective mass is determined to be $0.069 \pm 0.015 m_{\mathrm{e}}$, where $m_{\mathrm{e}}$ is the freeelectron mass. This effective mass measured by ARPES is different from the values reported by transport measurements ${ }^{13,14,16}, 0.052$ and $0.038 m_{\mathrm{e}}$ for electrons and holes respectively. This difference may be due to the fact that transport measurements are not momentum selective, and therefore the mass measured is the average mass over all $k_{z}$ values. On the other hand, ARPES is momentum selective and the value for the effective mass is only for this specific $k_{z}$ value. Taking this into account, the agreement between these measurements is reasonable. In summary, the data presented so far show that the low-energy excitations in graphite change from massless Dirac fermions with linear dispersion near $\mathrm{H}$ (Figs 1-3) to quasiparticles with parabolic dispersion and finite effective mass near K (Fig. 4a).

We now discuss another interesting feature observed in graphite, that is, a large electron pocket near $E_{\mathrm{F}}$. Figure $4 \mathrm{e}$ shows the intensity map measured in the same experimental conditions as Fig. $4 \mathrm{~d}$ except at a different spot. In Fig. 4e, a strong and large electron pocket within $50 \mathrm{meV}$ below $E_{\mathrm{F}}$ is the dominant feature. Weak signatures of the parabolic $\pi$ band (as in Fig. $4 \mathrm{~d}$ ) can still be observed, as the MDC at $E_{\mathrm{F}}$ (Fig. 4f) demonstrates. Here, in addition to the two main peaks (black arrows) corresponding to the electron pocket, a central weak peak (grey arrow) corresponding to the top of the parabolic band can also be distinguished. From the separation $\left(\approx 0.1 \AA^{-1}\right)$ between the two main peaks at $E_{\mathrm{F}}$, the electron concentration is determined to be $8.0 \pm 0.7 \times 10^{19} \mathrm{~cm}^{-3}$, which is an order of magnitude higher than the values reported ${ }^{13,14}$. Moreover, from the dispersion (Fig. 4e), the effective mass is extracted to be $0.42 \pm 0.07 m_{\mathrm{e}}$, which is also much larger than any mass reported by transport measurements ${ }^{13,14,16}$.

This large electron pocket is observed in most of the samples measured, and thus it represents an important feature associated with graphite. We note that a similar large electron pocket has been reported recently and proposed to be associated with either edge states or dangling bonds ${ }^{12}$. Here we provide detailed 

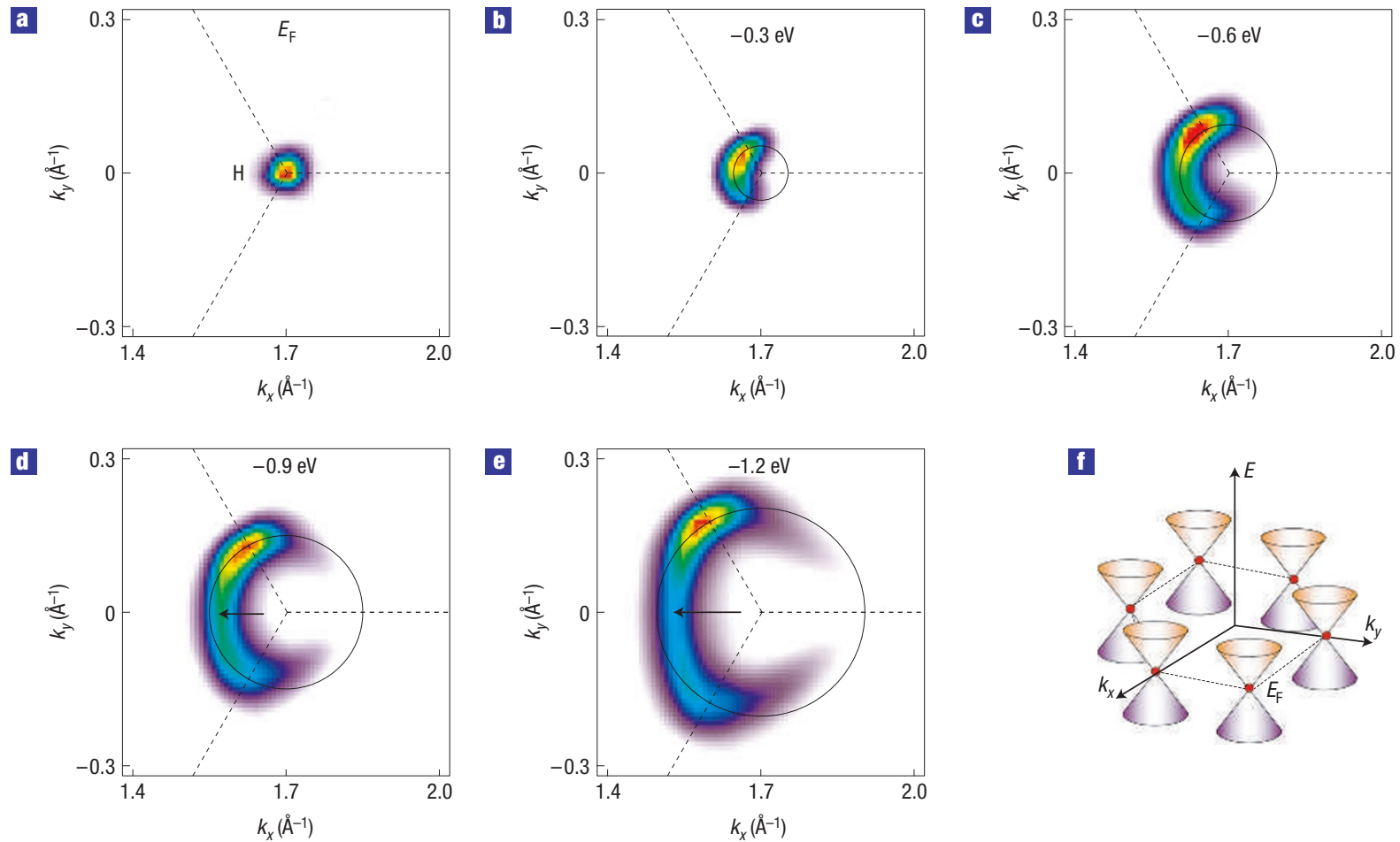

Figure 2 Constant energy maps taken near the $\mathrm{H}$ point, showing that the electronic structure is isotropic in the $k_{x}-k_{y}$ plane from $E_{\mathrm{F}}$ to $-0.6 \mathrm{eV}$. a-e, ARPES intensity maps near $\mathrm{H}\left(h v=140 \mathrm{eV}, k_{z} \approx 0.50 c^{*}\right)$ taken at energies from $E_{\mathrm{F}}$ to $-1.2 \mathrm{eV}$. The circles are guides for the circular intensity pattern near the $\mathrm{H}$ point. The arrows in $\mathbf{d}$ and e point to deviation from the circle. $\mathbf{f}$, Schematic diagram of the dispersion for graphene near six BZ corners in the three-dimensional $E-k_{x}-k_{y}$ space.
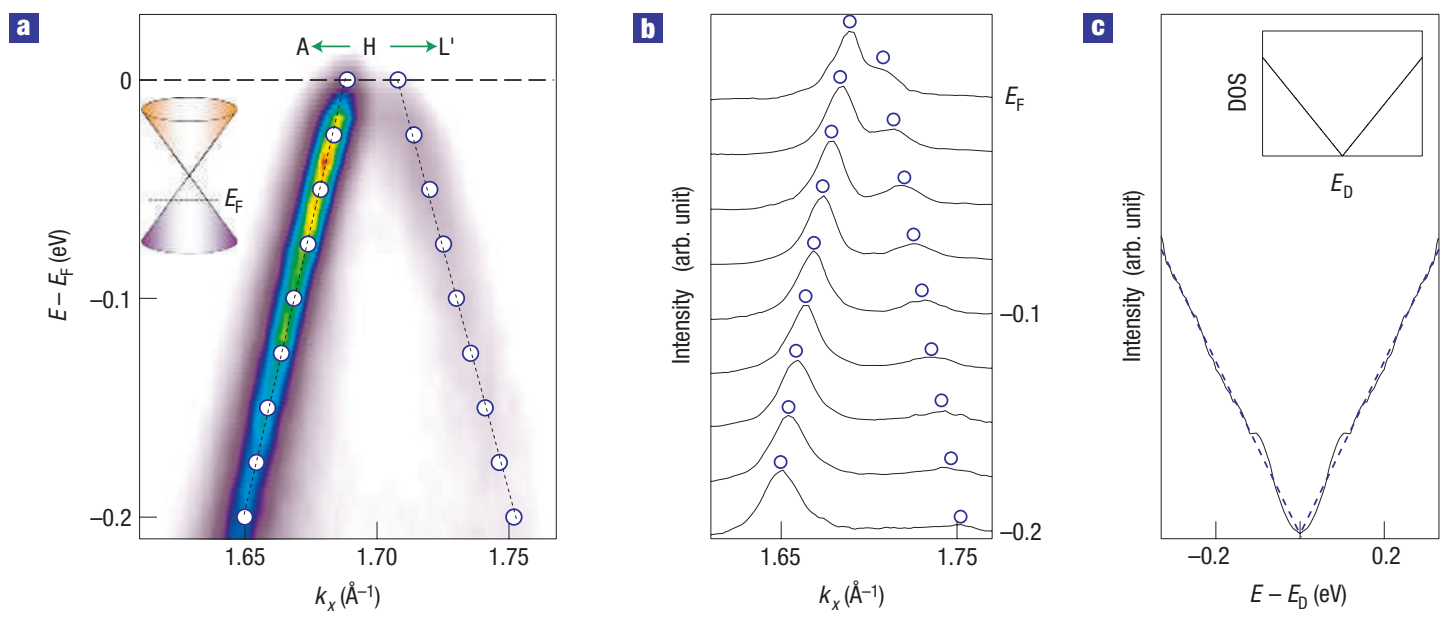

Figure 3 Detailed low-energy dispersion near the $\mathrm{H}$ point shows that low-energy excitations are Dirac fermions with the Dirac point slightly above $E_{\mathrm{F}}$. a, ARPES intensity map near the $\mathrm{H}$ point $\left(h v=65 \mathrm{eV}, k_{z} \approx 0.45 c^{*}\right)$ along the $\mathrm{AHL}$ ' direction (green line in the BZ shown in Fig. 1c). The open blue circles show the MDC dispersion. The dotted lines are guides for the linear dispersion. $\mathbf{b}, \mathrm{MDCs}$ at energies from $E_{\mathrm{F}}$ to $-0.2 \mathrm{eV}$ for the data shown in $\mathbf{a}$. Note that, similar to Fig. 2, the intensity of the $\pi$ band is strongly enhanced in the first $\mathrm{BZ}\left(\mathrm{H} \rightarrow \mathrm{A}\right.$ direction), due to the dipole matrix element ${ }^{10}$. c. Intensity obtained by integrating over both $k_{x}$ and $k_{y}$ for data taken near $\mathrm{H}$ $\left(h v=140 \mathrm{eV}, k_{z} \approx 0.50 c^{*}\right)$. The intensity has been symmetrized with respect to the Dirac point energy $\left(E_{\mathrm{D}} \approx 50 \mathrm{meV}\right)$ to compare directly with the expected density of states (DOS) for Dirac fermions (inset). An overall linear behaviour is observed with some weak additional intensity around 100 meV from the Dirac point energy. The origin of this additional weak intensity is unclear and needs further investigation.

characterization of this large electron pocket, which is important in revealing its origin. We propose defect-induced localized states as a possible explanation for this large electron pocket, on the basis of the following reasons. First, the electron concentration and effective mass measured for this electron pocket are much larger than reported values. Second, although the parabolic $\pi$ band in 

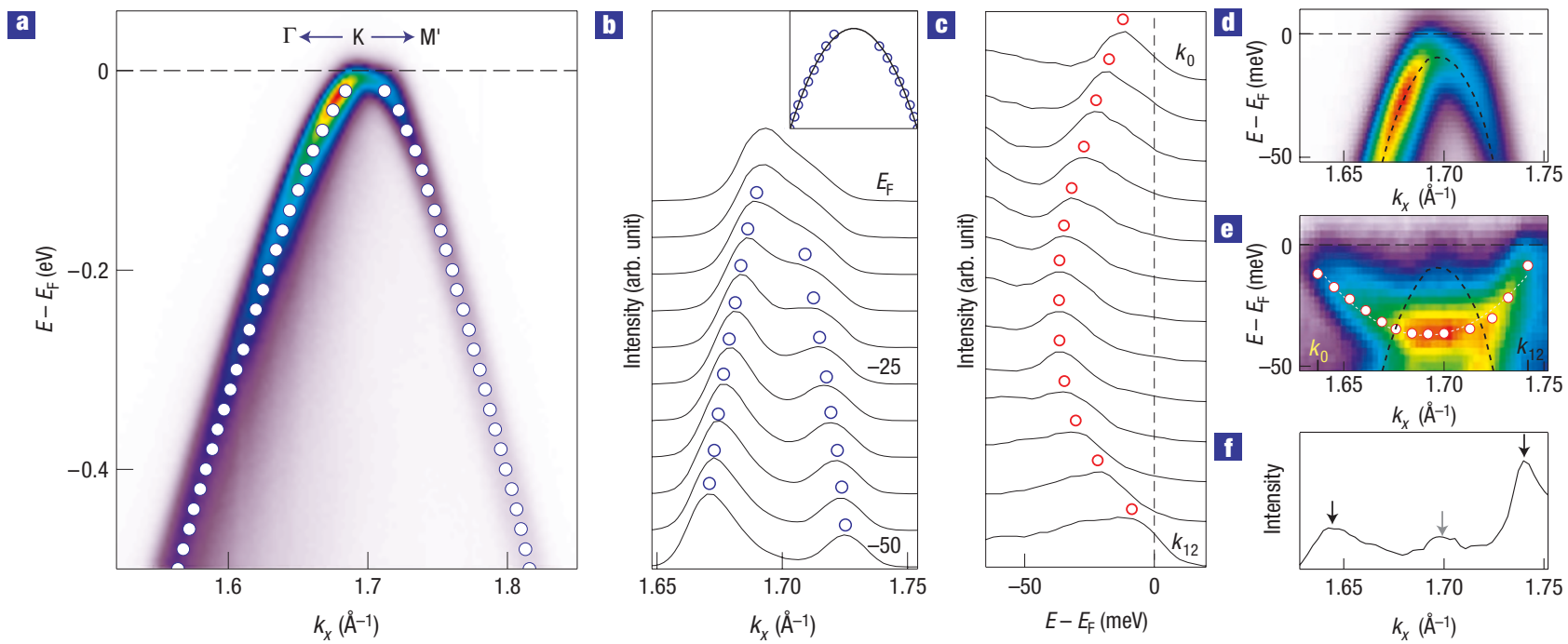

Figure 4 Detailed dispersion near $\mathrm{K}$, which shows that quasiparticles with finite effective mass and defect-induced localized states also contribute to the low-energy electronic dynamics. a, ARPES intensity map near $\mathrm{K}\left(h v=50 \mathrm{eV}, k_{z} \approx 0.08 c^{*}\right)$ along the $\Gamma \mathrm{KM}^{\prime}$ direction (blue line in the BZ shown in Fig. $\left.1 \mathrm{c}\right)$. The open circles are the dispersions extracted from MDCs. $\mathbf{b}, \mathrm{MDCs}$ from $E_{\mathrm{F}}$ to $-50 \mathrm{meV}$ for the data in $\mathbf{a}$. The open circles mark the peaks clearly resolved in the data. The inset shows the MDC dispersion from -10 to $-50 \mathrm{meV}$, with the parabolic fit used to extract the effective mass. c, EDCs from $k_{0}$ to $k_{12}$, as indicated in e. The open circles are the peak positions for the large electron pocket. $\mathbf{d}$, Closer view of the data shown in a with the EDC dispersion (dotted line). e, Intensity map near the $\mathrm{K}$ point measured in some parts of the sample, which shows an additional large electron pocket. The open circles are dispersions extracted from EDCs shown in $\mathbf{c}$. f, MDC at $E_{\mathrm{F}}$ from the data shown in e. The black arrows point to the peaks from the large electron pocket, which are separated by $\approx 0.1 \AA^{-1}$, whereas the grey arrow points to the peak from the $\pi$ band.
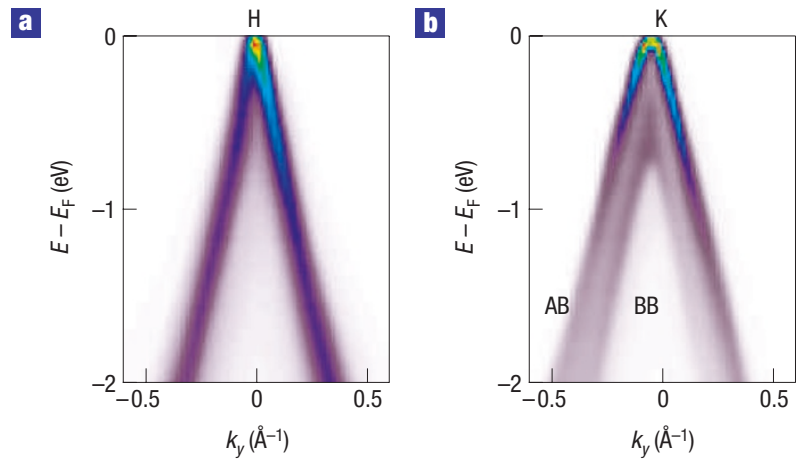

Figure 5 Dispersions measured near $\mathrm{H}$ and $\mathrm{K}$, showing the general consistency of the extracted $\boldsymbol{k}_{\boldsymbol{z}}$ values. a, Dispersion near $\mathrm{H}\left(h \nu=140 \mathrm{eV}, k_{z} \approx 0.50 c^{*}\right)$ along the $\mathrm{HH}^{\prime}$ direction, showing that the $\pi$ bands are degenerate. $\mathbf{b}$, Dispersion near $\mathrm{K}$ ( $h v=80 \mathrm{eV}, k_{z} \approx 0.07 c^{*}$ ) along the direction parallel to $\mathrm{HH}^{\prime}$, where the $\pi$ bands split into bonding (BB) and antibonding (AB) bands.

Fig. 4a,d is observed in all the samples measured and in different spots (averaged over $\approx 100 \mu \mathrm{m}$ ) within the same sample, this large electron pocket strongly depends on the spot position within the same sample. Third, scanning tunnelling microscopy shows that zigzag edges can induce a peak in the local density of states at an energy $(\approx-0.03 \mathrm{eV})$ similar to the electron pocket observed here ${ }^{17}$. In fact, it has been shown that a low concentration of defects (for example, edge states, vacancies and so on) can induce self-doping to the sample $e^{18,19}$. If this interpretation is correct, then further studies on this large electron pocket may provide insights into the magnetic properties of nano-graphite ribbons, because it has been proposed that some defect-induced localized states are magnetic ${ }^{20,21}$.

\section{METHODS}

High-resolution ARPES data have been taken at Beamlines 12.0.1 and 7.0.1 of the Advanced Light Source in Lawrence Berkeley National Laboratory with photon energies from 20 to $155 \mathrm{eV}$ with energy resolution from 15 to $65 \mathrm{meV}$. Data were taken from single-crystal graphite (Kish graphite for Fig. 3a,b and natural graphite for other figures) at a temperature of $25 \mathrm{~K}$. Throughout this paper, the $k_{z}$ values are estimated using the standard free-electron approximation of the ARPES final state ${ }^{22-24}$. The inner potential needed for extracting the $k_{z}$ value was determined from the periodicity of the detailed dispersion at the Brillouin zone centre $\Gamma A$ using a wide range of photon energies from 34 to $155 \mathrm{eV}$. The consistency of the $k_{z}$ values is confirmed by the degeneracy of the $\pi$ bands near $\mathrm{H}$ and a maximum splitting near $\mathrm{K}$ (Fig. 5), both in agreement with band structure ${ }^{11,25,26}$. Note that although this large energy range is good for discussing the splitting of the $\pi$ bands, it cannot capture the deviation from linear behaviour at the $\mathrm{K}$ point expected at low energy (as discussed in Fig. 4).

Received 18 April 2006; accepted 28 July 2006; published 27 August 2006.

References

1. Zhang, Y. B., Tan, Y.-W., Stormer, H. L. \& Kim, P. Experimental observation of the quantum Hall effect and Berry's phase in graphene. Nature 438, 201-204 (2005).

2. Novoselov, K. S. et al. Two-dimensional gas of Dirac fermions in graphene. Nature 438, 197-200 (2005).

3. Kopelevich, Y., Torres, J. H. S. \& da Silva, R. R. Reentrant metallic behavior of graphite in the quantum limit. Phys. Rev. Lett. 90, 156402 (2003).

4. Du, X., Tsai, S.-W., Maslov, D. L. \& Hebard, A. F. Metal-insulator-like behavior in semimetallic bismuth and graphite. Phys. Rev. Lett. 94, 166601 (2005).

5. Volovik, G. E. Field theory in superfluid ${ }^{3} \mathrm{He}$ : what are the lessons for particle physics, gravity and high-temperature superconductivity? Proc. Natl Acad. Sci. 96, 6042-6047 (1999).

6. Rantner, W. \& Wen, X.-G. Electron spectral function and algebraic spin liquid for the normal state of underdoped high $\mathrm{T}_{c}$ superconductors. Phys. Rev. Lett. 86, 003871 (2001).

7. Franz, M. \& Tesanovic, Z. Algebraic Fermi liquid from phase fluctuations: 'topological' Fermions, votex 'Berryons', and $\mathrm{QED}_{3}$ theory of cuprate superconductors. Phys. Rev. Lett. 87, 257003 (2001).

8. González, J., Guinea, F. \& Vozmediano, M. A. H. Unconventional quasiparticle lifetime in graphite. Phys. Rev. Lett. 77, 003589 (1996).

9. Luk'yanchuk, I. A. \& Kopelevich, Y. Phase analysis of quantum oscillations in graphite. Phys. Rev. Lett. 93, 166402 (2004)

10. Shirley, E. L., Terminello, L. J., Santoni, A. \& Himpsel, F. J. Brillouin-zone-selection effects in graphite photoelectron angular distributions. Phys. Rev. B 51, 013614 (1995).

11. Dresselhaus, M. S. \& Dresselhaus, G. Intercalation compounds of graphite. Adv. Phys. 51, $1-186(2002)$ 
12. Sugawara, K., Sato, T., Souma, S., Takahashi, T. \& Suematsu, H. Fermi surface and edge-localized states in graphite studied by high-resolution angle-resolved photoemission spectroscopy. Phys. Rev. B 73, 045124 (2006)

13. Soule, D. E. Magnetic field dependence of the Hall effect and magnetoresistance in graphite single crystals. Phys. Rev. 112, 698-707 (1958).

14. Zhang, Y. B., Small, J. P., Amori, M. E. S. \& Kim, P. Electric field modulation of galvanomagnetic properties of mesoscopic graphite. Phys. Rev. Lett. 94, 176803 (2005).

15. Toy, W. W., Dresselhaus, M. S. \& Dresselhaus, G. Minority carriers in graphite and the H-point magnetoreflection spectra. Phys. Rev. B 15, 4077-4090 (1977).

16. Galt, J. K., Yager, W. A. \& Dail, H. W. Jr. Cyclotron resonance effects in graphite. Phys. Rev. 103, 1586-1587 (1956).

17. Kobayashi, Y., Fukui, K.-I., Enoki, T., Kusakabe, K. \& Kaburagi, Y. Observation of zigzag and armchair edges of graphite using scanning tunneling microscopy and spectroscopy. Phys. Rev. B 71, 193406 (2005)

18. Peres, N. M. R., Guinea, F. \& Castro Neto, A. H. Electronic properties of disordered two-dimensional carbon. Phys. Rev. B 73, 125411 (2006).

19. Pereira, V. M., Guinea, F., Lopes Dos Santos, J. M. B., Peres, N. M. R. \& Castro Neto, A. H. Disorder induced localized states in graphene. Phys. Rev. Lett. 96, 036801 (2006).

20. Nakada, K., Fujita, M., Dresselhaus, G. \& Dresselhaus, M. S. Edge state in graphene ribbons: nanometer size effect and edge shape dependence. Phys. Rev. B 54, 017954 (1996).

21. Wakabayashi, K., Fujita, M., Ajiki, H. \& Sigrist, M. Electronic and magnetic properties of nanographite ribbons. Phys. Rev. B 59, 008271 (1999).

22. Law, A. R., Johnson, M. T. \& Hughes, H. P. Synchrontron-radiation-excited angle-resolved photoemission from single-crystal graphite. Phys. Rev. B 34, 004289 (1986)
23. Himpsel, F. J. Angle-resolved measurements of the photoemission of electrons in the study of solids. Adv. Phys. 32, 1-51 (1983).

24. Hüfner, S. Photoelectron Spectroscopy (Springer, Berlin, 1995).

25. Charlier, J.-C., Gonze, X. \& Michenaud, J.-P. First principles study of the electronic properties of graphite. Phys. Rev. B 43, 004579 (1991).

26. Nilsson, J., Castro Neto, A. H., Guinea, F. \& Peres, N. M. R. Electronic properties of graphene multilayers. Preprint at <http://www.arxiv.org/abs/cond-mat/0604106> (2006).

\section{Acknowledgements}

We thank A. Castro Neto, V. Oganesyan, A. Bill, K. McElroy, C. M. Jozwiak and D. Garcia for useful discussions and E. Domning and B. Smith for beam line 12.0.1 control software. This work was supported by the National Science Foundation through Grant No. DMR03-49361, the Director, Office of Science, Office of Basic Energy Sciences, Division of Materials Sciences and Engineering of the US

Department of Energy under Contract No. DEAC03-76SF00098 and by the Laboratory Directed

Research and Development Program of Lawrence Berkeley National Laboratory under the Department of Energy Contract No. DE-AC02-05CH11231.

Correspondence and requests for materials should be addressed to A.L.

Competing financial interests

The authors declare that they have no competing financial interests.

Reprints and permission information is available online at http://npg.nature.com/reprintsandpermissions/ 